\title{
Optimization of Phosphorus Fertilizer in Supplemental Feed-Fed Based Nile Tilapia (Oreochromis niloticus) Ponds
}

\author{
Moe Thidar Oo ${ }^{1}$, A M Shahabuddin ${ }^{1}$, Dhirendra $P$ Thakur ${ }^{1}$, Aye Aye Mon ${ }^{1}$, Amararatne \\ Yakupitiyage $^{1}$, Amrit N Bart ${ }^{1}$, Ram C Bhujel ${ }^{1} \&$ Jim S Diana ${ }^{2}$ \\ ${ }^{1}$ Aquaculture and Aquatic Resources Management, Asian Institute of Technology (AIT), Pathumthani, Thailand \\ ${ }^{2}$ School of Natural Resources and Environment, University of Michigan, Ann Arbor, MI, USA
}

Correspondence: A M Shahabuddin, Aquaculture and Aquatic Resources Management, Asian Institute of Technology (AIT), Pathumthani 12120, Thailand. E-mail: 513D3S2@m.mie-u.ac.jp

\begin{abstract}
An experiment was conducted in earthen ponds at the Asian Institute of Technology, Thailand to determine different phosphorus fertilizer dose effects on Nile tilapia production, water quality variables, nutrient utilization and cost-benefit under supplemental feeding. Five phosphorus fertilization rates were used as treatments e.g. 100\%, $75 \%, 50 \%, 25 \%$ and $0 \%$ of $7 \mathrm{~kg} \mathrm{P}$ ha week ${ }^{-1}$. Nitrogen fertilization rate was fixed at $28 \mathrm{~kg}$ $\mathrm{N}$ ha week ${ }^{-1}$ for all the treatments. Sex-reversed Nile tilapia were stocked at 3 fish m ${ }^{-2}$, and $30 \%$ CP floating feed fed at $50 \%$ satiation feeding rate. Nutrient budget showed higher phosphorus fertilizer input resulted in higher phosphorus sink in the sediment. Mean weight, mean weight gain, daily weight gain and net yield were not significantly different among treatments $(P>0.05)$. Total Kjeldahl nitrogen, total phosphorus and soluble reactive phosphorus were significantly different among treatments. Economic analysis showed phosphorus fertilization resulted in positive net returns. Though the gross income was not affected by different fertilization rates, significantly lowest cost was found in the treatment using $25 \%$ phosphorus fertilizer. It can be concluded from the research that $25 \%$ phosphorus fertilization might be used as an alternative strategy of Nile tilapia pond culture in terms of economic return and nutrient loss in sediment.
\end{abstract}

Keywords: phosphorus fertilizer, production, water quality, nutrient, Nile tilapia

\section{Introduction}

Nile tilapia (Oreochromis niloticus) is commonly produced in semi-intensive culture systems in Southeast Asia using fertilization to increase primary production (Boyd 1976; Diana, Lin \& Schneeberger 1991; FAO-FIES 2008; Asano, Hayashizaki, Eda, Khonglaliang \& Kurokura 2010). In many Asian countries, adding supplemental feed to fertilized ponds is becoming more and more popular for tilapia production. Supplemental feeding in fertilized ponds results in faster fish growth and higher pond yields at high stocking density compared with ponds receiving only fertilization (Hepher 1963; Tacon 1988). There is voluminous literature available on optimizing fertilization rate in fish ponds using inorganic or organic fertilizers or their combinations as the sole nutrient inputs (Boyd 1978; Olah 1986; Green, Teichert-Coddington \& Phelps 1990; Diana et al. 1991; Khud-Hansen, McNabb \& Batterson 1991; Edwards 1994; Lin, Teichert-Coddington, Green \& Vevrica 1997). The natural foods in fertilized ponds increase efficiency of supplemental feeds significantly and lead to lower feed conversion ration (FCR), and feeding rate of $50 \%$ ad libitum was optimal in ponds fertilized at a fixed rate of $28 \mathrm{~kg} \mathrm{~N}$ and $7 \mathrm{~kg}$ $\mathrm{P}$ ha week ${ }^{-1}$. Furthermore, it was observed that initiation of supplemental feeding at $50 \%$ ad libitum once fish reached $100 \mathrm{~g}$ is the most cost-effective way to produce large tilapia (Diana, Lin \& Yang 1996).

The above recommended fertilization rate is appropriate when fertilizer serves as the sole 
nutrient input for Nile tilapia culture in the tropics (Khud-Hansen et al. 1991). But, nutrients may become excessive in ponds with supplemental feeding, as substantial nutrients levels are also released from feeding wastes to pond water and eventually lead to excessive phytoplankton production (Lin 1990; Lin \& Diana 1995; Yi, Lin \& Diana 1996, 2001; Yi \& Lin 2001). It is ecologically and economically important to maintain adequate natural food production in fed ponds with balanced nutrient inputs from both external fertilization and internal wastes. To minimize nutrient waste, the rate of external fertilization needs to be adjusted according to the amount of nutrients derived from feeding waste. Such practice will result in more efficient nutrient utilization, better water quality, lower production cost and reduced nutrient load in pond effluents. Previous research has dealt with different fertilizers and feed combinations (Diana, Lin \& Jaiyen 1994; Milstein, Alkon, Karplus, Kochba \& Avnimelech 1995). However, almost all research to optimize supplemental feeding rate has been conducted in ponds with fixed fertilization rates, and none has been reported on optimizing fertilization regimes in fertilized ponds with supplemental feeds. Therefore, it was of interest to carry out the study to optimize external phosphorus fertilization rate for semi-intensive tilapia culture system under supplemental feeding. The purpose of the present study was to determine the phosphorus fertilization rate requirement for supplementary feed-fed Nile tilapia ponds.

\section{Materials and methods}

The experiment was carried out at the Asian Institute of Technology (AIT), Thailand for 130 day from September 2005 to January 2006. To avoid unwanted recruitment, all sex-reversed male Nile tilapia Oreochromis niloticus (average weight $100 \mathrm{~g}$ ) were purchased from AARM hatchery of AIT and were stocked in fifteen $200 \mathrm{~m}^{2}$ earthen ponds at 3 fish $\mathrm{m}^{-2}$. The experiment was conducted in a completely randomized block design. There were five treatments at different phosphorus fertilization rates with three replicates each. Phosphorus fertilizer (TSP- 46\% $\mathrm{P}_{2} \mathrm{O}_{5}$ ) rates for the five treatments were $0 \%, 25 \%, 50 \%, 75 \%$ and $100 \%$ of $7 \mathrm{~kg} \mathrm{P}$ ha week $^{-1}$ respectively. All ponds were fertilized with urea at $28 \mathrm{~kg} \mathrm{~N}$ ha week ${ }^{-1}$. Ponds were limed according to soil $\mathrm{pH}$ (Boyd 1995), and after 3 days of liming, ponds were filled and the water level was maintained at $1 \mathrm{~m}$ for the entire experiment. Ponds were fertilized for 2 weeks prior to fish stocking.

Tilapias in all experimental treatments were fed at feeding $50 \%$ ad libitum with floating complete pelleted feed (30\% crude protein). To determine feeding rate, fish were fed to satiation from 09:0010:00 hours and 15:00-16:00 hours once a week, and total consumption was determined for each pond. Average consumption for each treatment was used to set the feeding rate at $50 \%$ of that level for each treatment for the remainder of the week.

During the experiment, 30 fish were sampled every 2 weeks for batch weight and released back to ponds. Nutrient budget was calculated based on inputs and outputs of total nitrogen (TN) and total phosphorus (TP). At the beginning and the end of the experiment, water, fish and feed were sampled and analysed for moisture, TN and TP. Commercial inorganic fertilizers, urea $(46 \% \mathrm{~N})$ and TSP $\left(46 \% \mathrm{P}_{2} \mathrm{O}_{5}\right)$ were used and not analysed for $\mathrm{N}$ and $\mathrm{P}$ content. Initial and final soil samples (top $10 \mathrm{~cm}$ ) were collected from five locations in each pond using plastic tubes $(10 \mathrm{~cm}$ in length and $5 \mathrm{~cm}$ in diameter) and were analysed for bulk density, moisture, total nitrogen and total phosphorus. Pond water samples were taken biweekly at 09:00-09:30 hours using column sampler and analysed for total alkalinity, total ammonia nitrogen (TAN), nitrite nitrogen, nitrate nitrogen, total Kjeldahl nitrogen (TKN), soluble reactive phosphorus (SRP), total phosphorus (TP), chlorophyll $a$, total suspended solids (TSS) and total volatile solids (TVS) using standard methods (APHA 1985). Temperature, dissolved oxygen (DO) and $\mathrm{pH}$ (at $25 \mathrm{~cm}$ below water surface, middle and $25 \mathrm{~cm}$ above bottom) measured using a YSI model 58 oxygen meter, and Secchi disc visibility was measured in situ before collecting the water sample. In addition, monthly diurnal temperature, DO and pH were measured at 06:00, 10:00, 14:00, 18:00, 22:00 and 06:00 hours in each pond.

Growth performance of Nile tilapia was evaluated using the following calculations:

$$
\begin{aligned}
& \text { Feed Conversion ratio(FCR }) \\
& =\frac{\text { Weight of dry feed given }(\mathrm{g})}{\text { Wet weight gain }(\mathrm{g})}
\end{aligned}
$$

Daily Weight Gain $\left(\right.$ gday $\left.^{-1}\right)$

$$
=\frac{\text { Mean final weight }(\mathrm{g})-\text { Mean initial weight }(\mathrm{g})}{\text { Culture period }(\text { days })}
$$




$$
\text { Specific growth rate }(\%)=\frac{(\operatorname{Ln}(\text { Final mean weight })-\operatorname{Ln}(\text { Initial mean weight })) \times 100}{\text { Culture period }(\text { days })}
$$

Net yield $\left(\mathrm{kgm}^{-2}\right.$ per crop)

$$
\begin{gathered}
=\frac{\text { Final total weight }(\mathrm{kg})-\text { initial total weight }(\mathrm{kg})}{\text { Surface } \operatorname{areas}\left(\mathrm{m}^{2}\right)} \\
\text { Gross yield }\left(\mathrm{kgm}^{-2} \text { per crop }\right) \\
=\frac{\text { Final total weight }(\mathrm{kg})}{\text { Surface } \operatorname{areas}\left(\mathrm{m}^{2}\right)}
\end{gathered}
$$

Partial budget analysis was done based on farm gate prices in Thailand for harvested fish and current local market prices expressed in US dollar $(1 \mathrm{US}=39 \mathrm{Baht})$. Farm-gate wholesale price of Nile tilapia was fixed at $0.641 \mathrm{~kg}^{-1}$ for $300-400 \mathrm{~g}$ fish. Market price for juvenile sex-reversed Nile tilapia at 0.026 , urea at $0.178 \mathrm{~kg}^{-1}$, TSP at $0.298 \mathrm{~kg}^{-1}$, and feed at $0.48 \mathrm{~kg}^{-1}$ were applied to the analysis. To know the effect of different doses of fertilizer and block, data were analysed for significant differences among treatments using twoway analysis of variance (ANOVA) and regression using spss statistical software package. The significance of difference between mean was evaluated using Fisher's least significant difference (LSD), and differences among treatments were considered significant at an alpha level of 0.05. Means were given with \pm standard deviation (SD).

\section{Results}

\section{Supplemental feeding and phosphorus fertilizer input effect on growth}

Individual initial weight of Nile tilapia ranged from $93.2 \pm 0.2$ to $97.0 \pm 1.2 \mathrm{~g}$ in all treatments. At harvest, mean tilapia weight varied from $352.0 \pm 19.4$ to $375.6 \pm 10.1 \mathrm{~g}$, with daily mean weight gains $1.97 \pm 0.16-2.15 \pm 0.18 \mathrm{~g}$ fish day $^{-1}$ (Table 1). In general, high daily weight gain was registered in all treatments during the early experimental period, and a reduced daily weight gain was observed towards the end of the experiment. Growth performance of fish was positively correlated with feed input $(r=0.70$, $P<0.05$ ) (Figs 1 and 2). Total harvested biomass was not significantly different among treatments $(P>0.05)$. Survival rates ranged from 91 to $95 \%$ in all treatments. Highest gross yield was observed in highest phosphorus fertilizer input ponds. Highest net fish yields (156.42 \pm 13.29$)$ were observed in $100 \% \mathrm{P}$ and lowest were observed in $50 \% \mathrm{P}$ treatment $(P>0.05$, Table 1$)$.

Feed conversion ratio (FCR) ranged from $1.13 \pm 0.09$ to $1.24 \pm 0.06$ in all treatments, and was not significantly different among treatments $(P>0.05)$. Specific growth rates were not significantly different among treatments $(P>0.05)$.

\section{Effect of fertilizers on nutrient budgets of Nile tilapia ponds}

Nitrogen outputs in harvested fish and drained water were not significantly different among treatments $(P>0.05$, Table 2). Nutrient $(\mathrm{N}$ and $\mathrm{P})$ outputs in sediment were estimated by subtracting the total nutrient in the sediment before the experiment from total nutrients in the sediment at harvest, and thus, the gain of nutrients in the sediment in Tables 3 and 4 has been described as outputs. In all the treatments, urea fertilizer was the dominant of nitrogen source followed by feed, water and fish, whereas, feed was the main phosphorus input source followed by TSP fertilizer, stocked fish and water (Tables 3 and 4). Nitrogen outputs in sediment were significantly different between $100 \% \mathrm{P}$ and $0 \% \mathrm{P}$ treatments $(P<0.05)$. Lowest nitrogen deposition in sediment was observed in the treatment with 50\% P. However, nitrogen output in drain water was not significantly different among the treatments. Unaccounted nitrogen was highest in the treatment with $50 \% \mathrm{P}$ followed by $0 \%, 75 \%, 25 \%$ and $100 \%$ $\mathrm{P}(P<0.05$, Table 3$)$.

Phosphorus budgets revealed in treatments with $100 \% \mathrm{P}$ and $75 \% \mathrm{P}$ fertilizer was the dominant input ( $\mathrm{N}$ and $\mathrm{P}$ ) followed by feed. Phosphorus level in the inputs was significantly different among treatments $(P<0.05$, Table 4$)$. Phosphorus outputs in sediment were significantly higher in the treatments with $75 \%$ and $100 \% \mathrm{P}$ than those in treatments with $0 \%, 25 \%$ and 50\% $\mathrm{P}(P<0.05)$. Results showed highest phosphorus was deposited in the highest phosphorus loading rate compared with $25 \% \mathrm{P}$ and $0 \% \mathrm{P}$. Nutrients recovered in 
Table 1 Growth performance of Nile tilapia

\begin{tabular}{|c|c|c|c|c|c|}
\hline Parameters & $\begin{array}{l}\text { Treatment } 1 \\
(100 \% \mathrm{P})\end{array}$ & $\begin{array}{l}\text { Treatment } 2 \\
(75 \% \text { P) }\end{array}$ & $\begin{array}{l}\text { Treatment } 3 \\
\text { (50\% P) }\end{array}$ & $\begin{array}{l}\text { Treatment } 4 \\
(25 \% \mathrm{P})\end{array}$ & $\begin{array}{l}\text { Treatment } 5 \\
(0 \% \mathrm{P})\end{array}$ \\
\hline \multicolumn{6}{|l|}{ Stocking } \\
\hline Individual mean weight (g per fish) & $93.2 \pm 0.2$ & $97.0 \pm 1.2$ & $96.0 \pm 0.3$ & $95.8 \pm 1.4$ & $95.6 \pm 1.7$ \\
\hline Total weight (kg per pond) & $55.9 \pm 0.2$ & $58.2 \pm 0.7$ & $57.6 \pm 0.2$ & $57.5 \pm 0.8$ & $57.4 \pm 1.0$ \\
\hline Stocking density (fish $\mathrm{m}^{-2}$ ) & 3 & 3 & 3 & 3 & 3 \\
\hline \multicolumn{6}{|l|}{ Harvest } \\
\hline Individual mean weight (g per fish) & $372.4 \pm 23.3$ & $375.6 \pm 10.1$ & $353.7 \pm 35.0$ & $352.0 \pm 19.4$ & $355.3 \pm 25.9$ \\
\hline Total weight (kg per pond) & $212.3 \pm 13.4$ & $205.4 \pm 12.0$ & $191.8 \pm 11.6$ & $199.1 \pm 18.8$ & $199.7 \pm 13.1$ \\
\hline Survival rate $(\%)$ & $95 \pm 3$ & $91 \pm 8$ & $91 \pm 5$ & $94 \pm 4$ & $94 \pm 2$ \\
\hline FCR & $1.13 \pm 0.09$ & $1.17 \pm 0.10$ & $1.24 \pm 0.06$ & $1.18 \pm 0.11$ & $1.21 \pm 0.09$ \\
\hline Individual mean weight gain (g per fish) & $279.2 \pm 23.3$ & $278.5 \pm 9.6$ & $257.7 \pm 35.0$ & $256.2 \pm 20.6$ & $259.7 \pm 27.6$ \\
\hline Daily weight gain ( $\mathrm{g}$ fish day ${ }^{-1}$ ) & $2.15 \pm 0.18$ & $2.14 \pm 0.07$ & $1.98 \pm 0.27$ & $1.97 \pm 0.16$ & $1.99 \pm 0.21$ \\
\hline Specific growth rate $(\%)$ & $1.06 \pm 0.05$ & $1.04 \pm 0.02$ & $1.00 \pm 0.08$ & $1.00 \pm 0.05$ & $1.01 \pm 0.07$ \\
\hline Gross yield (kg per pond per crop) & $212.3 \pm 13.4$ & $205.4 \pm 12.0$ & $191.8 \pm 11.6$ & $199.1 \pm 18.8$ & $199.7 \pm 13.1$ \\
\hline Net fish yield (kg per pond per crop) & $156.42 \pm 13.29$ & $147.15 \pm 12.36$ & $134.23 \pm 11.45$ & $141.64 \pm 19.52$ & $142.35 \pm 14.02$ \\
\hline \multicolumn{6}{|l|}{ Recruitment } \\
\hline Total weight (kg per pond) & $9.7 \pm 4.06$ & $0.0 \pm 0.0$ & $2.4 \pm 1.2$ & $4.5 \pm 3.2$ & $3.0 \pm 4.2$ \\
\hline Mean weight (g per fish) & $13.2 \pm 5.0^{\mathrm{a}}$ & $0.0 \pm 0.0^{c}$ & $10.8 \pm 2.6^{\mathrm{ab}}$ & $7.5 \pm 4.8^{\mathrm{ac}}$ & $5.3 \pm 7.4^{\mathrm{bc}}$ \\
\hline
\end{tabular}

Values are mean $\pm \mathrm{SD}(n=3)$; mean values with different superscript letters in the same row were significantly different $(P<0.05)$.

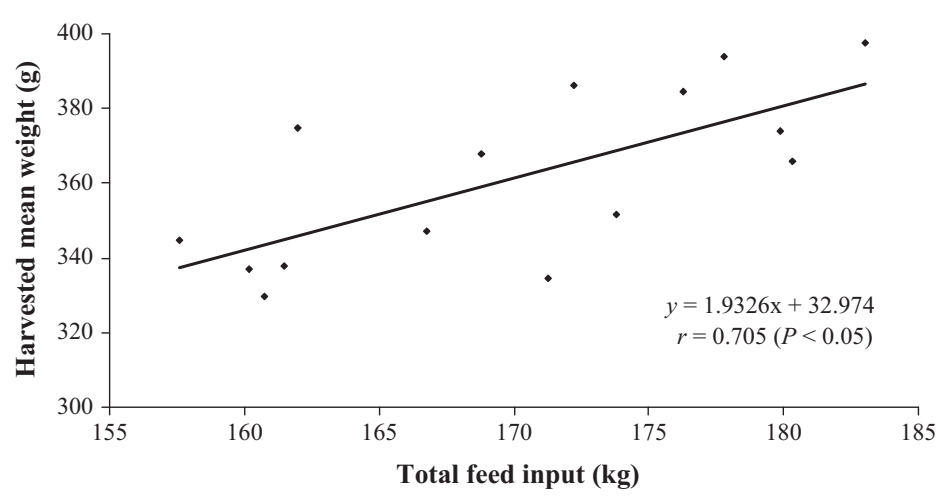

Figure 1 Relationship between harvested mean weights with total feed input. harvested fish biomass were $3.67 \pm 0.34,3.34 \pm$ $0.25, \quad 3.00 \pm 0.34, \quad 3.32 \pm 0.40$ and $3.33 \pm$ $0.18 \mathrm{~kg} \mathrm{~N}$ and, $0.81 \pm 0.12, \quad 0.78 \pm 0.06$, $0.76 \pm 0.22,0.79 \pm 0.11$ and $0.81 \pm 0.00 \mathrm{~kg} \mathrm{P}$ in the treatments with $100 \%, 75 \%, 50 \%, 25 \%$ and $0 \% \mathrm{P}$, respectively, and the values were not significantly different among treatments $(P>0.05$, Tables 3 and 4).

Fertilizer was the major nitrogen input source which accounted for 50.7-52.1\% total added nitrogen in all treatments. Percentage phosphorus input through feed increased significantly with reduced phosphorus fertilization rate. Feed was the only phosphorus input source in the treatment with $0 \% \mathrm{P}$, which accounted for $100 \%$ total phosphorus input (Table 5).
Percentage nitrogen gain in harvested biomass was not significantly different among treatments $(P>0.05)$. However, nitrogen gained in the sediment was significantly different among treatments, and 50\% $\mathrm{P}$ rate showed significantly lower phosphorus gained in the sediment compared with control $(P<0.05$, Table 5). Harvested fish gained $18.5-44.9 \%$ of the total added phosphorus inputs and was significantly higher in $0 \% \mathrm{P}$ than $25 \% \mathrm{P}$. Lowest phosphorus gained in biomass was observed in highest loading rate and was significantly lower than other treatments $(P<0.05)$. Nutrients trapped in sediment accounted for $34.9 \%$ to $73.7 \%$ total added nitrogen and 3.9$44.2 \%$ total added phosphorus; nitrogen percentage trapped in sediment was significantly different 
Figure 2 Relationship between net fish yield with total feed input.

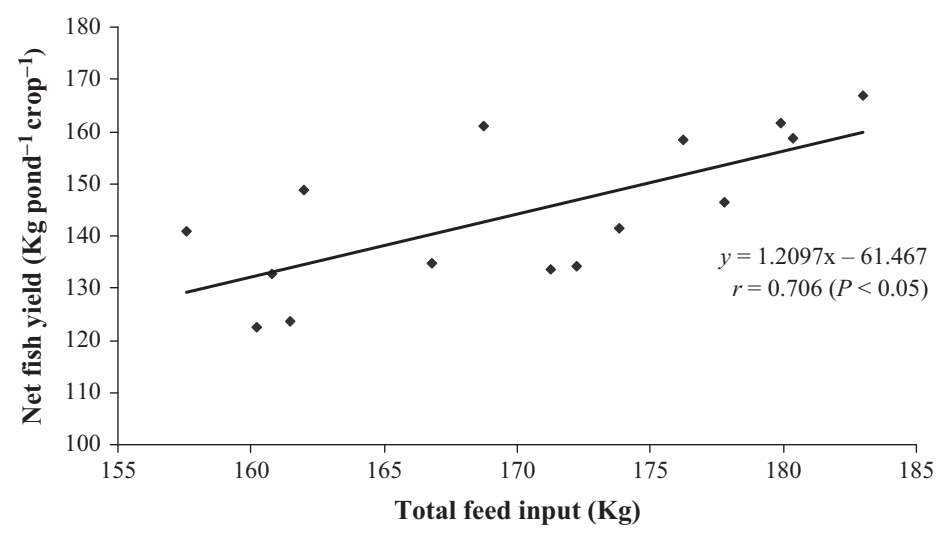

Table 2 Total nitrogen, total phosphorus and moisture contents (\%) of fish and sediment at harvest

\begin{tabular}{|c|c|c|c|c|c|}
\hline Parameters & $(100 \% \mathrm{P})$ & (75\% P) & $(50 \% \mathrm{P})$ & $(25 \% \mathrm{P})$ & $(0 \% \mathrm{P})$ \\
\hline \multicolumn{6}{|l|}{ Tilapia } \\
\hline Moisture & 71.33 & 70.69 & 70.75 & 69.55 & 69.93 \\
\hline TN (Dry wt) & 7.68 & 7.45 & 7.32 & 7.34 & 7.39 \\
\hline TP (Dry wt) & 1.70 & 1.73 & 1.79 & 1.73 & 1.77 \\
\hline \multicolumn{6}{|l|}{ Sediment } \\
\hline Moisture & 46.61 & 43.20 & 46.83 & 46.55 & 45.70 \\
\hline TN (Dry wt) & 0.18 & 0.18 & 0.18 & 0.17 & 0.18 \\
\hline TP (Dry wt) & 0.11 & 0.09 & 0.08 & 0.07 & 0.09 \\
\hline \multicolumn{6}{|l|}{ Recruitment } \\
\hline Moisture & 77.68 & - & 77.68 & 77.68 & 77.68 \\
\hline TN (Dry wt) & 9.26 & - & 9.26 & 9.26 & 9.26 \\
\hline TP (Dry wt) & 1.70 & - & 1.70 & 1.70 & 1.70 \\
\hline
\end{tabular}

between treatments with $100 \% \mathrm{P}$ and $50 \% \mathrm{P}$ $(P<0.05)$. There were significant differences in the phosphorus percentage trapped in sediments at $100 \%$ and $75 \%$, which were higher than other treatments $(P<0.05)$. Lowest phosphorus was trapped in the $25 \% \mathrm{P}$ rate.

\section{Effect of different phosphorus fertilizer doses on water quality parameters}

Mean temperatures in all the treatments ranged from 28.6 to $28.9^{\circ} \mathrm{C}$. Diurnal measurement showed mean early morning (6 am) DO in all treatments ranged from 0.79 to $0.93 \mathrm{mg} \mathrm{L}^{-1}$ $(P>0.05)$ but mean DO concentrations at 9 am in all treatments ranged from 1.92 to $2.58 \mathrm{mg} \mathrm{L}^{-1}$ and was significantly different among treatments $(P<0.05$; Table 6$)$. The value was highest in $100 \% \mathrm{P}$ treatment and lowest was in $0 \% \mathrm{P}$ treatment. Mean DO concentration in all treatments fluctuated between $1.00-4.70 \mathrm{mg} \mathrm{L}^{-1}$ during the experiment. The highest DO concentra- tion was recorded at the beginning of the experiment in all treatments. DO in all treatments showed declining trend with experiment progression, and dropped below $2.5 \mathrm{mg} \mathrm{L}^{-1}$ on the $8^{\text {th }}$ week. From $8^{\text {th }}$ week onwards, DO values showed relatively small fluctuation in all treatments and ranged between $1.00-3.00 \mathrm{mg} \mathrm{L}^{-1}$. $\mathrm{pH}$ ranged from 6.9 to 7.8 in all treatments during the experiment, and mean $\mathrm{pH}$ values were not significantly different among treatments $(P>0.05$; Table 6). Diurnal fluctuation of DO showed early morning level was critically lower in all treatments and increased sharply with time of day. Diurnal DO, $\mathrm{pH}$ and temperature reached highest levels at $2 \mathrm{pm}$ and then decreased gradually. Diurnal DO level decreased below $2 \mathrm{mg} \mathrm{L}^{-1}$ at $10 \mathrm{pm}$. The fluctuation of DO, $\mathrm{pH}$ and temperature in all treatments were shown correlation with time of day (Fig. 3). The mean values of total alkalinity ranged from 94 to $104 \mathrm{mg} \mathrm{L}^{-1}$ and its fluctuation showed a distinct but irregular trend over the experiment. Mean TAN values in all treatments 
Table 3 Total nitrogen budget (kg per pond)

\begin{tabular}{|c|c|c|c|c|c|}
\hline \multirow[b]{2}{*}{ Parameters } & \multicolumn{5}{|c|}{ Total nitrogen $(\mathbf{k g})$} \\
\hline & $\begin{array}{l}\text { Treatment } 1 \\
(100 \% \mathrm{P})\end{array}$ & $\begin{array}{l}\text { Treatment } 2 \\
(75 \% \mathrm{P})\end{array}$ & $\begin{array}{l}\text { Treatment } 3 \\
(50 \% \mathrm{P})\end{array}$ & $\begin{array}{l}\text { Treatment } 4 \\
(25 \% \mathrm{P})\end{array}$ & $\begin{array}{l}\text { Treatment } 5 \\
(0 \% \mathrm{P})\end{array}$ \\
\hline \multicolumn{6}{|l|}{ Inputs } \\
\hline Tilapia & $1.10 \pm 0.00$ & $1.15 \pm 0.02$ & $1.13 \pm 0.01$ & $1.13 \pm 0.02$ & $1.13 \pm 0.02$ \\
\hline Feed & $9.84 \pm 0.41$ & $9.63 \pm 0.52$ & $9.35 \pm 0.55$ & $9.30 \pm 0.69$ & $9.61 \pm 0.27$ \\
\hline Water & $1.00 \pm 0.12$ & $1.28 \pm 0.42$ & $1.68 \pm 0.45$ & $1.54 \pm 0.20$ & $1.91 \pm 0.56$ \\
\hline Fertilizer & $10.10 \pm 0.00$ & $10.10 \pm 0.00$ & $10.10 \pm 0.00$ & $10.10 \pm 0.00$ & $10.10 \pm 0.00$ \\
\hline Total & $22.04 \pm 0.46$ & $22.16 \pm 0.87$ & $22.26 \pm 0.37$ & $22.08 \pm 0.88$ & $22.75 \pm 0.31$ \\
\hline \multicolumn{6}{|l|}{ Outputs } \\
\hline Tilapia & $4.77 \pm 0.34$ & $4.48 \pm 0.24$ & $4.14 \pm 0.34$ & $4.45 \pm 0.39$ & $4.45 \pm 0.17$ \\
\hline Water & $1.89 \pm 0.15$ & $2.04 \pm 0.26$ & $1.76 \pm 0.28$ & $2.51 \pm 0.95$ & $2.05 \pm 0.51$ \\
\hline Sediment & $14.70 \pm 1.50^{\mathrm{a}}$ & $12.25 \pm 1.50^{\mathrm{ab}}$ & $6.87 \pm 4.66^{c}$ & $12.01 \pm 1.84^{\mathrm{ab}}$ & $7.91 \pm 3.02^{b c}$ \\
\hline Total & $21.33 \pm 1.15^{\mathrm{a}}$ & $18.80 \pm 1.67^{\mathrm{ab}}$ & $12.77 \pm 5.11^{\mathrm{c}}$ & $19.00 \pm 1.51^{\mathrm{ab}}$ & $14.40 \pm 3.64^{\mathrm{bc}}$ \\
\hline \multicolumn{6}{|l|}{ Gain } \\
\hline Tilapia & $3.67 \pm 0.34$ & $3.34 \pm 0.25$ & $3.00 \pm 0.34$ & $3.32 \pm 0.40$ & $3.33 \pm 0.18$ \\
\hline Water & $0.89 \pm 0.13$ & $0.75 \pm 0.67$ & $0.07 \pm 0.73$ & $0.97 \pm 1.02$ & $0.14 \pm 0.72$ \\
\hline Sediment & $14.70 \pm 1.50^{\mathrm{a}}$ & $12.25 \pm 1.50^{\mathrm{ab}}$ & $6.87 \pm 4.66^{\mathrm{c}}$ & $12.01 \pm 1.84^{\mathrm{ab}}$ & $7.91 \pm 3.02^{\mathrm{bc}}$ \\
\hline Unaccounted & $0.68 \pm 0.97^{c}$ & $3.39 \pm 2.49^{b c}$ & $9.51 \pm 4.91^{\mathrm{a}}$ & $3.10 \pm 2.41^{b c}$ & $8.33 \pm 3.48^{\mathrm{ab}}$ \\
\hline
\end{tabular}

Values are mean $\pm \mathrm{SD}(n=3)$; mean values with different superscript letters in the same row were significantly different $(P<0.05)$.

Table 4 Total phosphorus budget (kg per pond)

\begin{tabular}{|c|c|c|c|c|c|}
\hline \multirow[b]{2}{*}{ Parameters } & \multicolumn{5}{|c|}{ Total phosphorus (kg) } \\
\hline & $\begin{array}{l}\text { Treatment } 1 \\
(100 \% \mathrm{P})\end{array}$ & $\begin{array}{l}\text { Treatment } 2 \\
(75 \% \mathrm{P})\end{array}$ & $\begin{array}{l}\text { Treatment } 3 \\
(50 \% \mathrm{P})\end{array}$ & $\begin{array}{l}\text { Treatment } 4 \\
(25 \% \mathrm{P})\end{array}$ & $\begin{array}{l}\text { Treatment } 5 \\
(0 \% \mathrm{P})\end{array}$ \\
\hline \multicolumn{6}{|l|}{ Inputs } \\
\hline Tilapia & $0.25 \pm 0.00$ & $0.26 \pm 0.00$ & $0.26 \pm 0.00$ & $0.26 \pm 0.00$ & $0.26 \pm 0.00$ \\
\hline Feed & $1.83 \pm 0.08$ & $1.80 \pm 0.10$ & $1.76 \pm 0.10$ & $1.75 \pm 0.13$ & $1.80 \pm 0.05$ \\
\hline Water & $0.08 \pm 0.03$ & $0.07 \pm 0.04$ & $0.06 \pm 0.01$ & $0.06 \pm 0.02$ & $0.05 \pm 0.02$ \\
\hline Fertilizer & $2.52 \pm 0.00^{\mathrm{a}}$ & $1.91 \pm 0.00^{\mathrm{b}}$ & $1.26 \pm 0.00^{c}$ & $0.61 \pm 0.00^{d}$ & - \\
\hline Total & $4.67 \pm 0.06^{\mathrm{a}}$ & $4.03 \pm 0.06^{\mathrm{b}}$ & $3.33 \pm 0.06^{c}$ & $2.67 \pm 0.12^{d}$ & $2.10 \pm 0.00^{\mathrm{e}}$ \\
\hline \multicolumn{6}{|l|}{ Outputs } \\
\hline Tilapia & $1.06 \pm 0.12$ & $1.04 \pm 0.06$ & $1.02 \pm 0.23$ & $1.05 \pm 0.10$ & $1.06 \pm 0.01$ \\
\hline Water & $0.16 \pm 0.05$ & $0.14 \pm 0.04$ & $0.11 \pm 0.01$ & $0.12 \pm 0.03$ & $0.12 \pm 0.03$ \\
\hline Sediment & $1.92 \pm 0.13^{\mathrm{a}}$ & $1.48 \pm 0.71^{\mathrm{a}}$ & $0.42 \pm 0.35^{\mathrm{b}}$ & $0.09 \pm 0.04^{b}$ & $0.07 \pm 0.04^{\mathrm{b}}$ \\
\hline Total & $3.17 \pm 0.06^{\mathrm{a}}$ & $2.67 \pm 0.74^{\mathrm{a}}$ & $1.53 \pm 0.60^{\mathrm{b}}$ & $1.27 \pm 0.15^{\mathrm{b}}$ & $1.27 \pm 0.06^{b}$ \\
\hline \multicolumn{6}{|l|}{ Gain } \\
\hline Tilapia & $0.81 \pm 0.12$ & $0.78 \pm 0.06$ & $0.76 \pm 0.22$ & $0.79 \pm 0.11$ & $0.81 \pm 0.00$ \\
\hline Water & $0.08 \pm 0.03$ & $0.08 \pm 0.02$ & $0.05 \pm 0.02$ & $0.06 \pm 0.03$ & $0.06 \pm 0.02$ \\
\hline Sediment & $1.92 \pm 0.13^{\mathrm{a}}$ & $1.48 \pm 0.71^{\mathrm{a}}$ & $0.42 \pm 0.35^{\mathrm{b}}$ & $0.09 \pm 0.04^{b}$ & $0.07 \pm 0.04^{\mathrm{b}}$ \\
\hline Unaccounted & $1.55 \pm 0.11$ & $1.37 \pm 0.67$ & $1.79 \pm 0.58$ & $1.41 \pm 0.09$ & $0.86 \pm 0.07$ \\
\hline
\end{tabular}

Values are mean $\pm \mathrm{SD}(n=3)$; mean values with different superscript letters in the same row were significantly different $(P<0.05)$.

were from 0.83 to $1.97 \mathrm{mg} \mathrm{L}{ }^{-1}$. The TAN concentration in $0 \% \mathrm{P}$ treatment was higher than all other treatments during most samplings, the lowest mean was observed in highest phosphorus fertilizer ponds. Nitrate-N and nitrite-N in all treatments were from 1.43 to $1.99 \mathrm{mg} \mathrm{L}^{-1}$ and
0.31 to $0.53 \mathrm{mg} \mathrm{L}^{-1}$ respectively. Nitrate-N and nitrite-N fluctuated irregularly over the experiment. Mean nitrate- $\mathrm{N}$ and nitrite- $\mathrm{N}$ concentrations over the experiment were not significantly different among treatments $(P>0.05$; Table 6$)$. Mean TKN in all the treatments range from 3.53 to 
Table 5 Distribution (\%) of TN and TP in different treatments

\begin{tabular}{|c|c|c|c|c|c|}
\hline Parameters & $\begin{array}{l}\text { Treatment } 1 \\
(100 \% \mathrm{P})\end{array}$ & $\begin{array}{l}\text { Treatment } 2 \\
(75 \% \mathrm{P})\end{array}$ & $\begin{array}{l}\text { Treatment } 3 \\
(50 \% \mathrm{P})\end{array}$ & $\begin{array}{l}\text { Treatment } 4 \\
(25 \% \text { P) }\end{array}$ & $\begin{array}{l}\text { Treatment } 5 \\
(0 \% \mathrm{P})\end{array}$ \\
\hline \multicolumn{6}{|l|}{ Nitrogen } \\
\hline \multicolumn{6}{|l|}{ Inputs } \\
\hline Feed & $49.30 \pm 1.01$ & $48.77 \pm 1.31$ & $48.07 \pm 1.42$ & $47.90 \pm 1.84$ & $48.77 \pm 0.70$ \\
\hline Fertilizer & $50.70 \pm 1.01$ & $51.23 \pm 1.31$ & $51.93 \pm 1.42$ & $52.10 \pm 1.84$ & $51.23 \pm 0.70$ \\
\hline Total & 100.00 & 100.00 & 100.00 & 100.00 & 100.00 \\
\hline \multicolumn{6}{|l|}{ Gain } \\
\hline Tilapia & $18.40 \pm 1.66$ & $16.90 \pm 0.95$ & $15.43 \pm 1.50$ & $17.10 \pm 1.45$ & $16.87 \pm 0.97$ \\
\hline Water & $4.50 \pm 0.75$ & $3.87 \pm 3.50$ & $0.33 \pm 3.72$ & $5.07 \pm 5.35$ & $0.67 \pm 3.61$ \\
\hline Sediment & $73.70 \pm 6.78^{\mathrm{a}}$ & $62.23 \pm 9.16^{\mathrm{ab}}$ & $34.90 \pm 22.68^{c}$ & $62.10 \pm 11.30^{\mathrm{ab}}$ & $40.20 \pm 15.60^{b c}$ \\
\hline Total & $96.60 \pm 4.99^{a}$ & $83.00 \pm 12.49^{\mathrm{ab}}$ & $50.63 \pm 26.22^{c}$ & $84.27 \pm 11.66^{\mathrm{ab}}$ & $57.77 \pm 17.58^{\mathrm{bc}}$ \\
\hline Unaccounted & $3.40 \pm 4.99^{\circ}$ & $17.00 \pm 12.49^{b c}$ & $49.37 \pm 26.22^{\mathrm{a}}$ & $15.73 \pm 11.66^{\mathrm{bc}}$ & $42.23 \pm 17.58^{\mathrm{ab}}$ \\
\hline \multicolumn{6}{|l|}{ Phosphorus } \\
\hline \multicolumn{6}{|l|}{ Inputs } \\
\hline Feed & $42.06 \pm 0.99^{\mathrm{e}}$ & $48.51 \pm 1.34^{d}$ & $58.19 \pm 1.31^{\mathrm{c}}$ & $74.00 \pm 1.32^{b}$ & $100.00 \pm 0.00^{\mathrm{a}}$ \\
\hline Fertilizer & $57.94 \pm 0.99^{\mathrm{a}}$ & $51.49 \pm 1.34^{\mathrm{b}}$ & $41.81 \pm 1.31^{\mathrm{c}}$ & $26.00 \pm 1.32^{d}$ & $0.00 \pm 0.00^{e}$ \\
\hline Total & 100.00 & 100.00 & 100.00 & 100.00 & 100.00 \\
\hline \multicolumn{6}{|l|}{ Gain } \\
\hline Tilapia & $18.47 \pm 2.61^{\mathrm{c}}$ & $20.97 \pm 1.17^{c}$ & $25.17 \pm 7.27^{\circ}$ & $33.47 \pm 3.02^{b}$ & $44.90 \pm 1.05^{\mathrm{a}}$ \\
\hline Water & $1.80 \pm 0.61$ & $2.07 \pm 0.51$ & $1.60 \pm 0.75$ & $2.63 \pm 1.37$ & $3.30 \pm 0.95$ \\
\hline Sediment & $44.17 \pm 3.59^{a}$ & $39.50 \pm 18.02^{\mathrm{a}}$ & $13.77 \pm 11.68^{b}$ & $3.87 \pm 1.25^{\mathrm{b}}$ & $4.20 \pm 2.08^{b}$ \\
\hline Total & $64.47 \pm 1.79$ & $62.57 \pm 18.82$ & $40.53 \pm 19.41$ & $39.93 \pm 4.63$ & $52.37 \pm 3.15$ \\
\hline Unaccounted & $35.53 \pm 1.79$ & $37.43 \pm 18.82$ & $59.47 \pm 19.41$ & $60.07 \pm 4.63$ & $47.63 \pm 3.15$ \\
\hline
\end{tabular}

Values are mean $\pm \mathrm{SD}(n=3)$; mean values with different superscript letters in the same row were significantly different $(P<0.05)$.

Table 6 Water quality parameters measured biweekly

\begin{tabular}{|c|c|c|c|c|c|}
\hline Parameters & $\begin{array}{l}\text { Treatment } 1 \\
(100 \% \mathrm{P})\end{array}$ & $\begin{array}{l}\text { Treatment } 2 \\
(75 \% \text { P) }\end{array}$ & $\begin{array}{l}\text { Treatment } 3 \\
(50 \% \mathrm{P})\end{array}$ & $\begin{array}{l}\text { Treatment } 4 \\
(25 \% \mathrm{P})\end{array}$ & $\begin{array}{l}\text { Treatment } 5 \\
(0 \% \mathrm{P})\end{array}$ \\
\hline Temperature $\left({ }^{\circ} \mathrm{C}\right)$ & $28.6 \pm 0.1$ & $28.6 \pm 0.1$ & $28.9 \pm 0.3$ & $28.7 \pm 0.2$ & $28.8 \pm 0.3$ \\
\hline $\mathrm{DO}$ at $9 \mathrm{am}\left(\mathrm{mg} \mathrm{L}^{-1}\right)$ & $2.58 \pm 0.28^{\mathrm{a}}$ & $2.41 \pm 0.22^{\mathrm{ab}}$ & $2.33 \pm 0.14^{\mathrm{ab}}$ & $2.18 \pm 0.25^{\mathrm{bc}}$ & $1.92 \pm 0.09^{\mathrm{c}}$ \\
\hline $\mathrm{pH}$ & $7.4 \pm 0.1$ & $7.4 \pm 0.0$ & $7.4 \pm 0.0$ & $7.4 \pm 0.1$ & $7.3 \pm 0.1$ \\
\hline Secchi disc visibility $(\mathrm{cm})$ & $11 \pm 1$ & $11 \pm 3$ & $11 \pm 2$ & $11 \pm 0$ & $12 \pm 1$ \\
\hline Alkalinity $\left(\mathrm{mg} \mathrm{L}^{-1}\right)$ & $76 \pm 26$ & $94 \pm 14$ & $76 \pm 21$ & $96 \pm 4$ & $104 \pm 14$ \\
\hline TAN $\left(\mathrm{mg} \mathrm{L}^{-1}\right)$ & $0.83 \pm 0.58$ & $1.05 \pm 0.55$ & $1.12 \pm 0.91$ & $1.35 \pm 0.36$ & $1.97 \pm 1.07$ \\
\hline $\mathrm{NO}_{3}-\mathrm{N}\left(\mathrm{mg} \mathrm{L}^{-1}\right)$ & $1.69 \pm 0.84$ & $1.84 \pm 0.25$ & $1.44 \pm 0.45$ & $1.99 \pm 0.78$ & $1.43 \pm 0.70$ \\
\hline $\mathrm{NO}_{2}-\mathrm{N}\left(\mathrm{mg} \mathrm{L}^{-1}\right)$ & $0.43 \pm 0.05$ & $0.31 \pm 0.05$ & $0.38 \pm 0.10$ & $0.52 \pm 0.10$ & $0.53 \pm 0.11$ \\
\hline TKN (mg L $\left.{ }^{-1}\right)$ & $7.30 \pm 0.84^{b}$ & $7.89 \pm 0.78^{b}$ & $7.94 \pm 1.07^{\mathrm{b}}$ & $8.51 \pm 0.98^{\mathrm{ab}}$ & $9.49 \pm 1.94^{a}$ \\
\hline $\mathrm{TP}\left(\mathrm{mg} \mathrm{L}^{-1}\right)$ & $0.63 \pm 0.12^{\mathrm{a}}$ & $0.61 \pm 0.16^{\mathrm{a}}$ & $0.56 \pm 0.06^{\mathrm{ab}}$ & $0.54 \pm 0.10^{\mathrm{ab}}$ & $0.47 \pm 0.07^{\mathrm{b}}$ \\
\hline $\mathrm{SRP}\left(\mathrm{mg} \mathrm{L}^{-1}\right)$ & $0.03 \pm 0.02^{\mathrm{ab}}$ & $0.03 \pm 0.01^{\mathrm{bc}}$ & $0.03 \pm 0.02^{\mathrm{ab}}$ & $0.04 \pm 0.01^{\mathrm{a}}$ & $0.02 \pm 0.01^{c}$ \\
\hline TSS (mg L-1) & $188 \pm 21$ & $165 \pm 52$ & $149 \pm 18$ & $161 \pm 37$ & $130 \pm 31$ \\
\hline TVS (mg L $\left.{ }^{-1}\right)$ & $60 \pm 7$ & $54 \pm 12$ & $54 \pm 5$ & $57 \pm 3$ & $51 \pm 4$ \\
\hline Chlo-a $\left(\mu \mathrm{g} \mathrm{L}^{-1}\right)$ & $131 \pm 29$ & $132 \pm 44$ & $103 \pm 31$ & $114 \pm 30$ & $84 \pm 13$ \\
\hline
\end{tabular}

Values are mean $\pm \mathrm{SD}(n=3)$; mean values with different superscript letters in the same row were significantly different $(P<0.05)$.

$16.03 \mathrm{mg} \mathrm{L}^{-1}$. The TKN concentration in all treatments peaked around d 70 and thereafter showed a declining trend. The TKN concentration in $0 \% \mathrm{P}$ treatment was higher than all other treatments during most sampling and was significantly different than $100 \%, 75 \%$ and $50 \% \mathrm{P}$ over the experiment $(P<0.05$; Table 6$)$. Mean TP in all treatments ranged from 0.23 to $1.06 \mathrm{mg} \mathrm{L}^{-1}$, and TP concentration in all treatments increased linearly over the experiment except decline at the end of the experiment. Mean TP concentrations were significantly $(P<0.05)$ higher in treatment $100 \%$ 

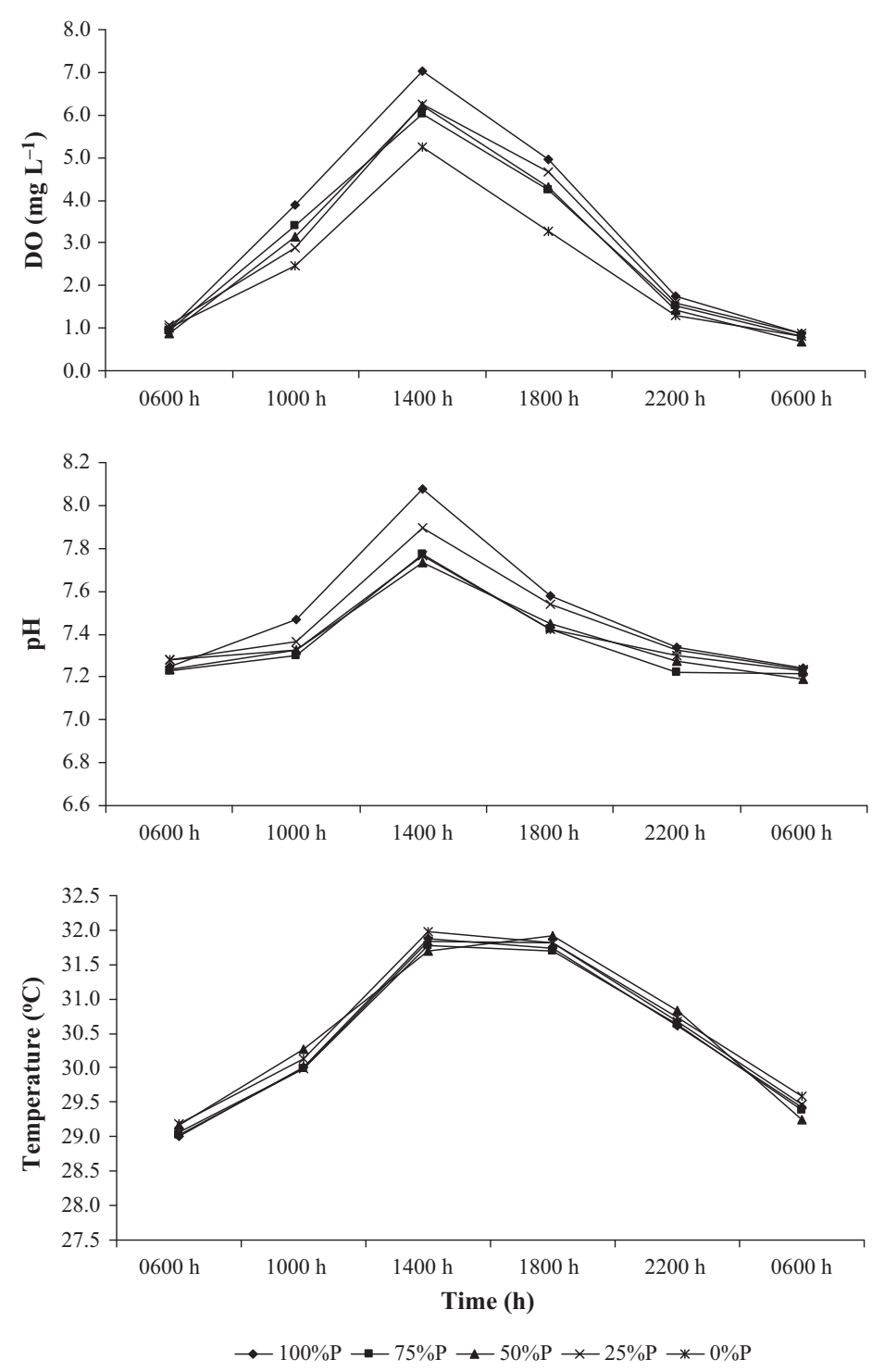

Figure 3 Fluctuations of mean $\mathrm{DO}, \mathrm{pH}$ and temperature in diurnal measurements.

$\mathrm{P}$ and $75 \% \mathrm{P}$ than $0 \% \mathrm{P}$ (Table 6), lowest value was observed in control. Soluble reactive phosphorus (SRP) concentrations in all treatments dropped throughout the experiment. There was a significant difference in mean SRP concentrations among treatments with significantly lower values found in $0 \% \mathrm{P}$ than other treatments except $75 \%$ $\mathrm{P}(P<0.05)$. Mean chlorophyll $a$ was not significantly different among treatments $(P>0.05$; Table 6). Chlorophyll $a$ fluctuated over the experiment. The highest chlorophyll $a\left(261.55 \mu \mathrm{g} \mathrm{L}^{-1}\right)$ concentration was observed in $100 \% \mathrm{P}$ treatment at the end of culture, and the lowest (13.33 $\mu \mathrm{g} \mathrm{L}^{-1}$ ) was observed in $0 \% \mathrm{P}$ at the beginning of culture. The lowest mean Chlorophyll a was observed also in $0 \%$ treatment. In general, chlorophyll a showed increasing trend over the culture period. Total suspended solids (TSS) and total volatile solid (TVS) increased in all treatments over the experiment. There were no significant differences in mean and final TSS and TVS values among all treatments $(P>0.05$; Table 6$)$. Mean and final Secchi disc depths were not significantly different among treatments. Total suspended solids (TSS) increased linearly with increasing chlorophyll a concentration. A positive correlation was also found between total volatile solid (TVS) and chlorophyll $a$ concentration during the experiment. Secchi disc visibility decreased linearly with increasing chlorophyll $a$ concentration. 


\section{Partial budget analysis to compare profit in different fertilizer doses}

Economic analysis indicated there were significant differences in total variable costs. The highest coast was observed in $100 \% \mathrm{P}$ treatment and lowest was found in $25 \% \mathrm{P}$ treatment. Gross revenue from tilapia and gross return varied among treatments. Highest cost of working capital was observed in $100 \% \mathrm{P}$ treatments and was significantly higher than $50 \%, 25 \%$ and $0 \%$ P treatment. (Table 7). Comparing cost of capital, total cost and gross return 25\% P treatment had comparatively higher gross returns than 75\%, 50\% and $0 \% \mathrm{P}$.

\section{Discussion}

Results of the study showed growth was correlated with supplemental feed and in different phosphorus fertilizer doses conditions were not strongly correlated. However, it was observed supplemental feeding with lower phosphorus fertilizer dose might be better for growth and culture environment.

In the present study, some of the physical, chemical and biological variables were different among treatments, indicating all treatments might not have similar culture environments. Tilapia growth rate observed in this study is similar to those (1.74-2.15 $\mathrm{g}$ fish day $\left.^{-1}\right)$ in fed alone ponds or fertilized ponds with supplemental feeding. Results of this study are comparable to growth rate observed under similar culture conditions for different nitrogen fertilization rates with supplemental feeding. However, a higher growth rate (2.66 $\mathrm{g}$ fish day $\left.^{-1}\right)$ of Nile tilapia under continued regular pond fertilization in addition to $50 \%$ satiation feeding was also reported. Daily weight gain in all treatments fluctuated during the experiment, but, in general, showed a declining trend with culture progress. Furthermore, the relatively slower growth tilapia during the experiment corresponds to the lower dissolve oxygen concentration. This result indicates low DO concentration during night and mainly in the early morning hours in all treatments, particularly towards the latter half of culture, might have slowed Nile tilapia growth and thus, masked the treatment effects in this study might be because of the fluctuation of DO in the ponds (Tsadik \& Kutty 1987).

In this study, feed conversion ratio was similar in all treatments and ranged from 1.13 to 1.24 . FCR observed in this study is similar to the previous report on optimum nitrogen rates (Truc 2005). However, a lower FCR (0.87) was reported in fertilized ponds throughout culture and feeding starting from day 80 (Thakur, Yi, Diana \& Lin 2004). Though there was no difference in FCR among treatments; lowest FCR was observed in the highest fertilizer treatment. Minimum fertilizer amount in supplemental feeding systems could improve water quality variables, might be feed intake in tilapia ponds was influenced by environmental factors (Zonneveld \& Fadholi 1991). Final tilapia mean weights and net yield were positively correlated with total feed inputs. The results of the

Table 7 Partial budget analysis to compare profit (US\$ $\mathrm{kg}$ ha week ${ }^{-1}$ )

\begin{tabular}{|c|c|c|c|c|c|}
\hline Parameters & $\begin{array}{l}\text { Treatment } 1 \\
(100 \% \mathrm{P})\end{array}$ & $\begin{array}{l}\text { Treatment } 2 \\
(75 \% \mathrm{P})\end{array}$ & $\begin{array}{l}\text { Treatment } 3 \\
(50 \% \mathrm{P})\end{array}$ & $\begin{array}{l}\text { Treatment } 4 \\
(25 \% \mathrm{P})\end{array}$ & $\begin{array}{l}\text { Treatment } 5 \\
(0 \% \mathrm{P})\end{array}$ \\
\hline \multicolumn{6}{|l|}{ Gross revenue } \\
\hline Tilapia & $136.10 \pm 8.58$ & $131.63 \pm 7.71$ & $122.97 \pm 7.44$ & $127.63 \pm 12.02$ & $128.07 \pm 8.43$ \\
\hline \multicolumn{6}{|l|}{ Variable cost } \\
\hline Seed cost & $15.6 \pm 0.0$ & $15.6 \pm 0.0$ & $15.6 \pm 0.0$ & $15.6 \pm 0.0$ & $15.6 \pm 0.0$ \\
\hline Feed cost & $91.23 \pm 3.75$ & $89.30 \pm 4.81$ & $86.80 \pm 5.03$ & $86.40 \pm 6.36$ & $89.30 \pm 2.45$ \\
\hline Urea & $3.9 \pm 0.0$ & $3.9 \pm 0.0$ & $3.9 \pm 0.0$ & $3.9 \pm 0.0$ & $3.9 \pm 0.0$ \\
\hline TSP & $3.80 \pm 0.00^{\mathrm{a}}$ & $2.80 \pm 0.00^{b}$ & $1.90 \pm 0.00^{\circ}$ & $0.90 \pm 0.00^{d}$ & - \\
\hline Cost of working capital & $3.27 \pm 0.12^{\mathrm{a}}$ & $3.17 \pm 0.15^{\mathrm{ab}}$ & $3.07 \pm 0.12^{b}$ & $3.07 \pm 0.21^{b}$ & $3.10 \pm 0.10^{b}$ \\
\hline Total cost & $117.73 \pm 3.85^{\mathrm{a}}$ & $114.83 \pm 4.96^{\mathrm{ab}}$ & $111.23 \pm 5.17^{b}$ & $109.80 \pm 6.53^{b}$ & $111.90 \pm 2.55^{b}$ \\
\hline Gross return & $18.37 \pm 8.03$ & $16.80 \pm 7.33$ & $11.73 \pm 3.67$ & $17.83 \pm 7.41$ & $16.13 \pm 6.33$ \\
\hline Added cost & 5.85 & 2.93 & -0.67 & -2.07 & - \\
\hline Added return & 2.23 & 0.69 & -4.40 & 1.68 & - \\
\hline Added return/added cost & 0.38 & 0.24 & 6.59 & -0.81 & - \\
\hline
\end{tabular}

Values are mean $\pm \mathrm{SD}(n=3)$; mean values with different superscript letters in the same row were significantly different $(P<0.05)$. 
study indicate that under supplementary feeding conditions, growth performance of Nile tilapia was dependent on the added feed followed by the nutrient inputs.

Nutrient budgets revealed feed and fertilizer were the greatest nitrogen sources and phosphorus inputs. Supplemental feeds and fertilizers are the predominant nutrient inputs among various sources in closed ponds (Edwards 1992; Lin, Yi \& Diana 1997).

The nutrient recovery harvested fish (15.43$18.40 \%$ ) in the present study was similar to those (15.45-20.04\%) reported previously (Lin, Teichert-Coddington, Green \& Vevirica 1996). However, higher nitrogen recovery (19.26-37.14\%) was observed in harvested fish in an experiment on optimum nitrogen rates (Truc 2005). Phosphorus recovery percentage (18.47-44.90\%) in fish harvested in the present study was higher than those (10.02-15.10\%) reported previously (Lin et al. 1996). Nitrogen budgets showed a large portion of the total nitrogen inputs (34.90-73.70\% N) went to the sediment. In the case of phosphorus, highest phosphorus in fish body was recovered in treatment 25\% P and lowest in $100 \% \mathrm{P}$ treatment. However, a large proportion of total nutrient inputs $(35.53-60.07 \%$ P) went unaccounted. The unaccounted phosphorus might have resulted from mud adsorption, as mud has strong attraction for phosphorus (Boyd 1985). Higher proportion of total phosphorus outputs in sediment was observed in the treatments with $100 \%$ and $75 \% \mathrm{P}$ than the other treatments, and lowest was observed in $25 \% \mathrm{P}$ treatment. This result indicates increased phosphorus fertilization rate resulted in higher phosphorus accumulation in the sediment. Similar findings showed that much phosphorus fertilizer is not used in the pond, rather than accumulated in the sediment (Masuda \& Boyd 1994). Nitrogen fertilizer not removed in fish was incorporated in sediment as organic nitrogen or lost to the atmosphere through denitrification and ammonia volatilization (Gross, Boyd \& Wood 2000). In the present study, nutrients contained in sediment were higher than those in the effluent water. The results are in agreement with the previous observation where they found that sediment layer of few centimetres deep contains more nutrient than the water column (Avnimelech, McHenry \& Ross 1984). Unnecessary fertilizer can be harmful as well as increase production costs. It was observed from this experiment that using of $25 \% \mathrm{P}$ fertilizer was the optimum dose for supplemental feed based earthen Nile tilapia ponds.

Water temperature during the experiment was suitable for tilapia feeding and growth (Chervinski 1982). Mean morning DO concentrations observed in this study were much higher than those reported previously (Truc 2005). The highest value was observed in the highest fertilizer loading treatment. The lowest was observed in $0 \% \mathrm{P}$ fertilizer treatment indicating phosphorus fertilizer is correlated with pond ecosystem nutrient dynamics. Moreover, low growth of tilapia in this study, observed during the latter half of culture, was correspondent with the low DO concentrations. It was also reported that when fish fed ad libitum, the weight gain and feed consumption decline with decreasing dissolved oxygen level (Boyd 1990). The TP and SRP were significantly lower in $0 \% \mathrm{P}$ treatment $(P<0.05)$. On the other hand, TKN was significantly higher in the same treatment. This indicates without phosphorus fertilizer, nitrogen fertilizer cannot be fully utilized by the pond ecosystem dynamics. Combination of both nitrogen and phosphorus in appropriate doses is the best for fish culture ponds.

However, other water quality variables were not significantly different among the treatments. In the present study, phosphorus concentration in water was not high even in the pond with the highest fertilizer rate. There was no significant difference in phosphorus concentration using lower phosphorus fertilizer dose compared with the highest dose $(P>0.05)$. The results showed higher phosphorus fertilizer rates do not necessarily result in higher phosphorus content in water column as the added phosphorous is removed from the water column by other processes; possibly sequestered in bottom sediment. Recently, in a study on bluegill, also observed higher rates of fertilizer had no effect on concentration of total nitrogen and phosphorus in pond water (Wudtisin \& Boyd 2005). Aquaculture ponds receiving feeds often have total phosphorus concentrations above $0.5 \mathrm{mg} \mathrm{L}^{-1}$ and total nitrogen concentrations of 2 or $3 \mathrm{mg} \mathrm{L}^{-1}$ (Boyd \& Tucker 1998). Total phosphorus and nitrogen concentrations in water in the present study during most of the sampling remained $0.5 \mathrm{mg} \mathrm{L}^{-1}$ and $4 \mathrm{mg} \mathrm{L}^{-1}$ respectively. Mean chlorophyll $a$ concentration in all treatments increased gradually over the experiment. Similar types of changes in primary production were previously observed when fertilization and feeding were done (Green 
1992). Total suspended solids (TSS) and total volatile solids (TVS) were positively correlated with the chlorophyll a concentration. The secchi disc visibility negatively correlated with chlorophyll $a$ concentration, indicating the number of phytoplankton increased with the time of experiment. The results in this study, indicate the majority of the pond turbidity is from phytoplankton and not from clay turbidity.

The economic analysis showed positive gross return was found in all treatments. Production of treatment $25 \% \mathrm{P}$ was higher than the treatment $50 \% \mathrm{P}$ and $75 \% \mathrm{P}$. It was reported that net revenue reached $\$ 5,029$ ha year $^{-1}$ when fish were fed at $50 \%$ satiation, starting feeding at $100 \mathrm{~g}$ in fertilized ponds, which was highest net revenue compared with fertilized ponds and feed alone ponds (Yi, Lin \& Diana 2002). Feed costs as a per cent of total costs averaged $77.49 \%$ to $79.80 \%$ for all treatments. Total production costs for the $100 \% \mathrm{P}$ was higher than $50 \% \mathrm{P}, 25 \% \mathrm{P}$ and $0 \% \mathrm{P}$ treatments. In this experiment, income from the sale of harvested fish was similar for all treatments. Among all treatments, $25 \% \mathrm{P}$ of standard phosphorus application ( $7 \mathrm{~kg} \mathrm{P}$ ha week ${ }^{-1}$ ) showed feed and fertilizer costs about $78.69 \%$ and $4.37 \%$, respectively of total production costs, which was the lowest among the treatments. It can be concluded from the research using 25\% P together with nitrogen fertilizer is suitable for supplemental feed-fed based Nile tilapia ponds.

\section{Acknowledgments}

The authors express deepest gratitude to Dr Yang Yi (deceased) for his valuable guidance, encouragement, great patience, suggestion and advice throughout the experiment. The author sincerely acknowledges the support from Aquaculture Collaborative Research Support Program (A/CRSP) for proving the scholarship and research funds to make this study possible.

\section{References}

APHA (1985) Standard Methods for the Examination of Water and Waster Water (15th edn) American Water Works Association and Water Pollution Control Federation, American public health association, Washington DC.

Asano Y., Hayashizaki K., Eda H., Khonglaliang T. \& Kurokura H. (2010) Natural foods utilized by Nile tila- pia Oreochromis niloticus in fertilizer based fish ponds in Lao PDR identified through stable isotope analysis. Fisheries science 76, 811-817.

Avnimelech Y., McHenry J.R. \& Ross D.J. (1984) Decomposition of organic matter in lake sediments. Environmental science and technology 18, 5-11.

Boyd C.E. (1976) Nitrogen fertilizer effects on production of tilapia in ponds fertilized with phosphorus and potassium. Aquaculture 7, 385-390.

Boyd C.E. (1978) Effluents from catfish ponds during fish harvest. Journal of Environmental quality 7, 59-62.

Boyd C.E. (1985) Chemical budget of channel catfish ponds. Transactions of the American Fisheries Society 114, 291-298.

Boyd C.E. (1990) Water Quality in Ponds for Aquaculture. Alabama Agricultural Experimental station, Auburn University, Alabama.

Boyd C.E. (1995) Bottom Soils. Sediment and Pond Aquaculture, Chapman and Hall, New York.

Boyd C.E. \& Tucker C.S. (1998) Pond Aquaculture Water Quality Management. Kluwer Academic Publishers, Boston, MA.

Chervinski J. (1982) Environmental physiology of tilapia. In: The Biology and Culture of Tilapia (ed. by R.S.V. Pullin \& R.H. Lowe-Mcconnell). International Center for Living Aquatic Resources Management (ICLARM), Manila.

Diana J.S., Lin C.K. \& Schneeberger P.J. (1991) Relationships among nutrient inputs, water nutrient concentrations, primary production and yield of Oreochromis niloticus in ponds. Aquaculture 92, 323-341.

Diana J.S., Lin C.K. \& Jaiyen K. (1994) Supplemental feeding of tilapia in fertilized ponds. Journal of the World Aquaculture Society 25, 497-506.

Diana J.S., Lin C.K. \& Yang Y. (1996) Timing of supplemental feeding for tilapia production. Journal of the world aquaculture society 27, 410-419.

Edwards P. (1992) Reuse of Human Wastes in Aquaculture: A Technical Review. Water and sanitation report No.2 World Bank, Washington DC.

Edwards P. (1994) An assessment of the role of buffalo manure for pond culture of tilapia. I. On-station experiment. Aquaculture 126, 83-95.

FAO-FIES (2008) Fishery and Aquaculture Statistics, 2006 , Book, IO400/T. Food and Agriculture Organization (FAO), Rome.

Green B.W. (1992) Substitution of organic manure for pelleted feed in tilapia production. Aquaculture 101, 213-222.

Green B.W., Teichert-Coddington D.R. \& Phelps R.P. (1990) Response of tilapia yield and economics to varying rates of organic fertilization and season in two Central American countries. Aquaculture 90, 279-290.

Gross A., Boyd C.E. \& Wood C.W. (2000) Nitrogen transformations and balance in channel catfish ponds. Aquaculture Engineering 24, 1-14. 
Hepher B. (1963) The years of research in fish pond fertilization in Israel. II. Fertilizer dose and frequency of fertilization. Bamidgeh 15, 78-92.

Khud-Hansen C.F., McNabb C.D. \& Batterson T.R. (1991) Application of Limnology for Efficient Nutrient Utilization in Tropical Pond Aquaculture. International Association of Theoretical and Applied Limnologists, Stuttgart.

Lin C.K. (1990) Integrated culture of walking catfish (Clarias macrocephalus) and tilapia (Oreochromis niloticus). In: Proceedings of the Second Asian Fisheries Forum (ed. by R. Hirano \& I. Hanyu). Asian Fisheries Society, Manila.

Lin C.K. \& Diana J.S. (1995) Coculture of catfish (Clarias macrocephalus x C. gariepinus) and tilapia (Oreochromis niloticus) in ponds. Aquatic Living Resources 8, 449-454.

Lin C.K., Teichert-Coddington D., Green B. \& Vevirica K. (1996) Fertilization Regimes, Agricultural and Aquatic Systems Program. Asian Institute of Technology, Bangkok.

Lin C.K., Teichert-Coddington D.R., Green B.W. \& Vevrica K.L. (1997) Fertilizer regimes. In: Dynamics of Pond Aquaculture (ed. by H.S. Egna \& C.E. Boyd). CRC Press, Florida.

Lin C.K., Yi Y. \& Diana J.S. (1997) The effects of pond management strategies on nutrient budgets: Thailand. In: Fourteenth Annual Technical Report (ed. by D. Burke, B. Goetze \& H.S. Egna). Pond Dynamics/Aquaculture, Collaborative Research Support Program, Oregon State University, Oregon.

Masuda K. \& Boyd C.E. (1994) Phosphorus fractions in soil and water of aquaculture ponds built on clayey. Journal of the world aquaculture society 25, 379-395.

Milstein A., Alkon A., Karplus I., Kochba M. \& Avnimelech Y. (1995) Combined effects of fertilization rate, manuring and feed pellet application on fish performance and water quality in polyculture ponds. Aquaculture research 26, 55-65.

Olah J. (1986) Carp production in manured ponds. In: Aquaculture of Cyprinids (ed. by R. Billard \& J. Marech). IRNA, Paris.

Tacon A.G.J. (1988) The nutrition and feeding of farmed fish and shrimp-A training manual: 3. Feeding methods. In: The Second International Symposium of Tilapia in Aquaculture (ed. by R.S.V. Pullin, T. Bhukaswan, K. Tonguthai \& I.L. Maclean), p. 15. International
Center for Living Aquatic Resources Management, Penang.

Thakur D.P., Yi Y., Diana J.S. \& Lin C.K. (2004) Effects of fertilization and feeding strategy on water quality, growth performance, nutrient utilization and economic return in Nile tilapia (Oreochromis niloticus) ponds. In: Proceedings of 6th International Symposium of Tilapia in Aquaculture (ed. by R. Bolivar, G. Mair \& K. Fitzsimmons). Pond dynamics/Aquaculture, Collaborative research support program, Oregon state university, Oregon.

Truc L.T.T. (2005) Optimization of a Nitrogen Fertilization Regime in Fertilized Nile Tilapia (Oreochromis niloticus) Ponds with Supplemental Feed. Asian Institute of Technology, Bangkok.

Tsadik G.G. \& Kutty M.N. (1987) Influence of Ambient Oxygen on Feeding and Growth of the Tilapia, Oreochromis niloticus (Linnaeus). Food and agricultural organization (FAO), Rome.

Wudtisin W. \& Boyd C.E. (2005) Determination of the phosphorus fertilization rate for bluegill ponds using regression analysis. Aquaculture research 36, 593-599.

Yi Y. \& Lin C.K. (2001) Effects of biomass of caged Nile tilapia (Oreochromis niloticus) and aeration on the growth and yields of all Nile tilapia in an integrated cage-cum-pond system. Aquaculture 195, 253-267.

Yi Y., Lin C.K. \& Diana J.S. (1996) Influence of Nile tilapia (Oreochromis niloticus) stocking density in cages on their growth and yield in cages and in ponds containing the cages. Aquaculture 146, 205-215.

Yi Y., Lin C.K. \& Diana J.S. (2001) Integrated recycle system for catfish and tilapia culture. In: Eighteenth Annual Technical Report- CRSP (ed. by A. Gupta, K. McElwee, D. Burke, J. Burright, X. Cummings \& H. Egna). Pond Dynamics/Aquaculture, Collaborative research support program, Oregon State University, Oregon.

Yi Y., Lin C.K. \& Diana J.S. (2002) Hybrid catfish (Clarias macrocephalus x C. gariepinus) and Nile tilapia (Oreochromis niloticus) culture in an integrated pen - cum-pond system: growth performance and nutrient budgets. Aquaculture 217, 395-408.

Zonneveld N. \& Fadholi R. (1991) Feed intake and growth of red tilapia at different stocking densities in ponds in Indonesia. Aquaculture 99, 83-94. 